\title{
Study of the Lorentz force on debris with high
}

\section{area-to-mass ratios}

\author{
Romain Serra ${ }^{\mathrm{a}}$ and Massimiliano Vasile ${ }^{\mathrm{b}}$ \\ University of Strathclyde, Glasgow, United Kingdom, G1 1 XJ \\ Kento Hoshi ${ }^{\mathrm{c}}$ and Hiroshi Yamakawa ${ }^{\mathrm{d}}$ \\ Kyoto University, Uji City, Kyoto Prefecture, Japan, 611-0011
}

The presence of plasma in Low Earth Orbit and above can be the cause of electrostatic charging on space objects with a conductive surface, which then become subject to the Lorentz force induced by the magnetic field. This paper investigates it effects on the trajectory of orbital debris with a high area-to-mass ratio, as their course is particularly influenced by non-gravitational perturbations such as atmospheric drag or solar radiation pressure. Depending on the altitude (low or medium) and the available data, different charging models have been coupled with an orbit propagator, featuring a range of non-Keplerian accelerations and both three and six degrees-of-freedom dynamics. In particular, there has been a focus on long-term effects by simulating charged versus non-charged objects over the time span of years or decades.

\footnotetext{
${ }^{\text {a }}$ Research Associate, Mechanical and Aerospace Department, 75 Montrose St.

b Professor, Mechanical and Aerospace Department, 75 Montrose St.

c PhD Student, Research Institute for Sustainable Humanosphere, Gokasho.

d Professor, Research Institute for Sustainable Humanosphere, Gokasho.
} 


\section{Nomenclature}

$$
\begin{aligned}
& a=\text { semi-major axis, } \mathrm{km} \\
& i=\text { inclination, } \operatorname{deg} \\
& \Omega=\text { right ascension of the ascending node, deg } \\
& \omega=\text { argument of perigee, deg } \\
& n=\text { mean motion, } \mathrm{s}^{-1} \\
& \mu=\text { planetary gravitation constant, } \mathrm{m}^{3} / \mathrm{s}^{2} \\
& m=\text { mass, } \mathrm{kg} \\
& s=\text { shadow function } \\
& \rho=\text { atmospheric density, } \mathrm{kg} / \mathrm{m}^{3} \\
& A=\text { area, } \mathrm{m}^{2} \\
& q=\text { electrostatic charge, Coulomb } \\
& C=\text { electrostatic capacitance, farad } \\
& \phi=\text { electrostatic potential, Volt } \\
& I=\text { surface current, Ampere } \\
& T=\text { temperature, } \mathrm{K} \\
& n_{\mathrm{s}}=\text { number density of species } \mathrm{s}, \mathrm{m}^{-3} \\
& k_{B}=\text { Boltzmann constant, } \mathrm{J} / \mathrm{K} \\
& \varepsilon=\text { permittivity, } \mathrm{F} / \mathrm{m} \\
& e=\text { elementary charge, Coulomb } \\
& Q=\text { quaternion } \\
& \mathbf{r}=\text { position, } \mathrm{km} \\
& \mathbf{v}=\text { velocity, } \mathrm{km} / \mathrm{s} \\
& \mathbf{I}=\text { inertia matrix }, \mathrm{m}^{2} / \mathrm{kg} \\
& \mathbf{B}=\text { magnetic field, Tesla }
\end{aligned}
$$

\section{Introduction}

Objects on orbit around the Earth can acquire a surface charge when going through space plasma. This situation occurs at low altitude in the ionosphere or even higher in the plasmasphere. 
Effects on trajectories are possible as the Earth's magnetic field affects the motion of electrostatic charges. This action is described in the magnetic component of the so-called Lorentz force. It is for instance causing ions to gyrate and drift in the Earth's magnetosphere. Similarly, the motion of dust particles around Jupiter can be significantly influenced by its powerful magnetic field [1]. Thus it is interesting to study how the Lorentz force impacts the orbit of space debris with a high area-to-mass ratio, which can be defined as larger than $0.1 \mathrm{~m}^{2} / \mathrm{kg}$. Typical examples are fragments of solar panels or pieces of thermal blanket, products of collisions. They are much more sensitive than other objects to non-gravitational forces such as atmospheric drag or solar radiation pressure. In a space environment known to be dynamically chaotic [2], their long-term behavior is quite different from the rest of the population and has been the subject of several studies $[3,4]$.

It is worth mentioning that the magnetic force has been considered for the design of space trajectories in the literature $[5,6]$, but these techniques require an artificial charging of the spacecraft in order for the control law to be significant. While the effects of natural surface charging on hardware are fairly well documented [7], its impact on spacecraft dynamics is less clear. The effects of the magnetic force on the evolution of Keplerian coordinates both in Low Earth Orbit (LEO) and Geostationary Earth Orbit (GEO) has been investigated in [8], including objects with high area-to-mass ratios. The main restriction was that orbit propagation was performed only over one period so that no long-term trend could be identified. A preliminary study by the authors probed this area by simulating trajectories over years in LEO as well as in Medium Earth Orbit (MEO), with the inclusion of a six degrees-of-freedom analysis [9]. The present paper extends it with an improved dynamical model.

This article is organized as follows. Section II introduces the integrators and dynamics adopted in this work, including a general description of the magnetic force. Sections III describes in details the charging model used in the Low Earth Orbit (LEO) and Medium Earth Orbit (MEO). Finally, simulations and analysis of the results are provided for these two environments in Section IV.

\section{Orbit propagation}

Computations of space trajectories are performed via the interface between an numerical inte- 
grator and a dynamical model, referred to as a propagator, all coded in $\mathrm{C}++$. When the Lorentz force is enabled, a wrapper is used to call an external Fortran implementation of the IRI-2012 model (available at http://irimodel.org). Assuming rigid bodies, spherical objects are simulated with three degrees of freedom (dof) whereas non-spherical geometries require six. The attitude and orbital mechanics are introduced after a description of the integration methods.

\section{A. Numerical integration}

For 3dof simulations, the motion of the object's barycenter is propagated with a set of orbital elements in the Earth-centered Inertial (ECI) frame. To avoid singularities, the modified equinoctial coordinates [10] are preferred over the Keplerian ones. For 6dof, the position-velocity vector is used instead, because of its closer proximity in frequency of oscillations with the attitude variables, which are represented here with quaternions. The numerical integrator used in this study is a 8 th order multi-step predictor-corrector known as Adam-Bashforth-Moulton (ABM) 8. It requires to be initialized, which is done here with a Bulirsh-Stoer algorithm of same order, but once running it requires only two evaluations of the dynamical model per step, which is particularly convenient given the high computational cost of IRI-2012. Note that the use of a symplectic integrator, better designed for long-term propagation than ABM, is impossible here due the magnetic force that cannot be derived from a potential.

\section{B. Dynamics}

The physical model exploited to propagate trajectories of space debris is described thereafter. Transformations between ECI and Earth-centered Earth-fixed (ECEF) are performed taking into account precession and nutation as well as the Earth rotation as reported in [11]. Apart from the

magnetic force itself, three others are considered here: the gravitation (Earth, Moon and Sun), the aerodynamics and the radiation pressure. 


\section{Forces}

In order to integrate the dynamics for both 3 and 6 dof models, the total perturbing accelerations acting on the center of mass of the object have to be computed. In what follows the model of perturbations considered in this study are detailed.

a. Gravity. In addition to the Keplerian term, the gravitational dynamics considered here takes into account high order terms in the Earth's potential as well as lunisolar perturbations. Hence the sum of accelerations due to gravity is:

$$
\begin{array}{r}
\ddot{\mathbf{r}}_{\mathrm{g}}=-\nabla U_{\mathrm{E}}+\mu_{\text {Sun }}\left(\frac{\mathbf{r}_{\text {Sun }}-\mathbf{r}}{\left|\mathbf{r}_{\text {Sun }}-\mathbf{r}\right|^{3}}-\frac{\mathbf{r}_{\text {Sun }}}{\left|\mathbf{r}_{\text {Sun }}\right|^{3}}\right)+ \\
\mu_{\text {Moon }}\left(\frac{\mathbf{r}_{\text {Moon }}-\mathbf{r}}{\left|\mathbf{r}_{\text {Moon }}-\mathbf{r}\right|^{3}}-\frac{\mathbf{r}_{\text {Moon }}}{\left|\mathbf{r}_{\text {Moon }}\right|^{3}}\right),
\end{array}
$$

where $\mathbf{r}$ stands for the position of an object, $\mu$ for the gravitational constant of a body and $U_{\mathrm{E}}$ for the classic expansion of the geopotential in spherical harmonics. Positions of the Sun and the Moon as functions of time are computed from low-precision ephemerides [11]. Note that the gradient in Eq. (1) is computed in the body-fixed frame before being translated in ECI. It is remarkable that the total acceleration due to gravity does not depend on the mass $m$ of the object itself.

b. Magnetic force. The acceleration due to the Lorentz force induced by an ambient magnetic field $\mathbf{B}$ on a charged object with a velocity $\mathbf{v}$ with respect to that field writes:

$$
\ddot{\mathbf{r}}_{L}=\frac{q}{m} \mathbf{v} \times \mathbf{B}
$$

where $q$ is the total electrostatic charge. It is important to note that the magnitude of this perturbation depends on the angle between the magnetic field and the relative velocity. In particular, it vanishes when they are aligned.

The computation of the Lorentz force is twofold. On the one hand, one needs to know the magnetic field $\mathbf{B}$ as well as the relative velocity $\mathbf{v}$ in order to compute their cross product. On the other hand, it requires the evaluation of the electric charge $q$, that depends on the spacecraft and the conditions of the ambient plasma. Details on how to compute it are given in Section III.

The expression of the Earth's magnetic field can be derived from the magnetic potential $V$ with the relation $\mathbf{B}=-\nabla V$. The potential is classically described via spherical harmonics expressed in 
spherical coordinates $(r, \Phi, \lambda)$ :

$$
V=R_{\mathrm{E}} \sum_{n=1}^{+\infty} \sum_{m=0}^{n}\left(\frac{R_{\mathrm{E}}}{r}\right)^{n+1}\left(g_{n}^{m} \cos (m \Phi)+h_{n}^{m} \sin (m \Phi)\right) P_{n}^{m}(\sin (\lambda)),
$$

where $R_{E}$ is the Earth's equatorial radius, the $P$ are the Schmidt quasi-normalized associated Legendre functions whereas $h$ and $g$ are the Gauss coefficients for the Earth's magnetic potential [12]. Note that Eq. (3) allows one to derive the Earth magnetic field in the Earth-Centered EarthFixed (ECEF) reference frame, then the velocity in Eq. (2) has to be expressed in ECEF and the resulting acceleration needs to be converted back to ECI.

c. Aerodynamics. The acceleration due to atmospheric drag writes:

$$
\ddot{\mathbf{r}}_{\mathrm{drag}}=-\frac{1}{2} C_{D} \frac{A}{m} \rho\left\|\mathbf{v}_{\mathrm{rel}}\right\|^{2} \frac{\mathbf{v}_{\mathrm{rel}}}{\left\|\mathbf{v}_{\mathrm{rel}}\right\|}
$$

where $\rho$ is the atmospheric density, $C_{D}$ the drag coefficient and $\mathbf{v}_{\text {rel }}$ the relative velocity with respect to the flow. Here the air density is computed from the Jacchia-Gill model [11]. As for the relative velocity, the atmosphere is considered as co-rotating with the Earth.

For 3dof propagations, it is enough to use only this term by setting the drag coefficient to 2 . For non-spherical objects, one needs to consider the three components of the aerodynamic forces: lift, drag and side. Here it is done by replacing the product $-C_{D} \frac{\mathbf{v}_{\text {rel }}}{\left\|\mathbf{v}_{\text {rell }}\right\|}$ in Eq. (4) with a function of attitude. To be more precise, the quaternions are converted into attack and side-slip angles which are then used as inputs for an aerodynamics database interpolated with the nearest neighbor approach. This database was derived from Direct Simulation Monte Carlo (DSMC) [13] performed on a $1 \times 1 \times 0.01 \mathrm{~m}$ object in a free molecular regime as described in [14].

d. Solar radiation pressure. The acceleration due to solar radiation writes:

$$
\ddot{\mathbf{r}}_{\mathrm{SRP}}=-\frac{A}{m} s C_{R} P_{\mathrm{Sun}} \frac{A U^{2}}{\left\|\mathbf{r}_{\text {Sun }}\right\|^{3}} \mathbf{r}_{\text {Sun }}
$$

where $C_{R}$ is the reflective coefficient, $A U$ is the astronomical unit, $\mathbf{r}_{\text {Sun }}$ the Sun's position (computed from ephemerides), $s$ the so-called shadow function and $P_{\text {Sun }}$ the solar radiation pressure in the vicinity of the Earth $\left(4.560 \times 10^{-6} \mathrm{~N} / \mathrm{m}^{2}\right)$.

The solar radiation pressure decreases in penumbra until it vanishes in umbra. The model presently adopted considers only the Earth as an occulting body. As described in [11], the function 
$s$ is computed as the area of the solar disk visible to the spacecraft, normalized so that its value is always between 0 and 1 .

\section{Torques}

For 6 dof propagations, it is necessary to compute the sum of moments acting on the barycenter.

a. Gravity gradient. The variations of the gravity field along the object are responsible for a torque. Here only the first order approximation of this effect for the Keplerian term is taken into account. It writes:

$$
\mathbf{M}_{g g}=\frac{\mu_{\mathrm{E}}}{\left\|\mathbf{r}_{G}\right\|^{3}} \mathbf{r}_{G} \times\left(\mathbf{I} \cdot \mathbf{r}_{G}\right)
$$

where the position of the barycenter relative to the Earth's center $\mathbf{r}_{G}$ is written in a frame fixed to the object where the inertia matrix is $\mathbf{I}$.

b. Aerodynamics. The three components of the aerodynamic torque on the center of symmetry are computed similarly to the corresponding force that is through the interpolation of a DSMC database taking attitude as input.

c. Other. For symmetrical geometries, the Lorentz force does not introduce any torque at first order on the center of symmetry as the surface charges are spread evenly over the object. It is the case for classic shapes (sphere, plate, etc.), as long as the surface is made of a single type of conductor, which is assumed to be the case in this study. The same rule applies for radiation pressure, assuming that the reflective properties of the surface are uniform. However, torques can be induced on the barycenter $\mathrm{G}$ if the latter is displaced with respect to the center of symmetry $\mathrm{S}$. In this case, the resulting moment $\mathbf{M}$ on $\mathbf{G}$ from a force $\mathbf{F}$ writes:

$$
\mathbf{M}=\mathbf{r}_{G S} \times \mathbf{F},
$$

where $\mathbf{r}_{G S}$ is the vector going from $\mathrm{G}$ to $\mathrm{S}$.

\section{Spacecraft charging}

This Section first describes in details the computations necessary to estimate the electrostatic charge of a space object in LEO. Then, a simplistic extension is proposed for the MEO environment 
in order to be able to perform simulations there too.

\section{A. In Low Earth Orbit}

As described in the previous section, the total electrostatic charge $q$ on the surface affects the magnetic force. When the object has a surface potential $\phi$, the total amount of surface charge $q$ writes:

$$
q=C \phi
$$

where $C$ is the electrostatic capacitance of the object. Thus these two quantities are needed to estimate the charge.

\section{Surface potential}

When a space object gets charged, various currents flow on its surface. In equilibrium, for the sake of continuity, the so-called current-balance equation must be satisfied [15]:

$$
I_{e}(\phi)-I_{i}(\phi)-I_{p h}(\phi)-I_{s e}(\phi)=0
$$

where $I_{e}$ denotes the ambient electron current, $I_{i}$ denotes the ambient ion current, $I_{p h}$ denotes the photoelectron current, and $I_{s e}$ denotes the secondary electron current (it includes a backscatter current and an elastically reflected current).

In a dense plasma such as in LEO, $I_{s e}$ is not significant because these current densities are relatively small $[16,17]$ with comparison to the ambient plasma current density, so the currentbalance equation can be written as:

$$
I_{e}(\phi)-I_{i}(\phi)-I_{p h}(\phi)=0
$$

In general, Equation (10) cannot be solved analytically and a numerical solver is needed. Here, a Newton-Raphson method is used. The details on how to compute the terms in the balance equation are given next.

a. Ambient plasma currents Current collection of ambient electron and ions by a charged object can be described the Orbital Motion Limitation (OML) theory, which was first proposed by 
Mott-Smith and Langmuir [18]. According to the current OML theory [19, 20], the ambient electron current for the Maxwellian distribution is

$$
I_{e}(\phi)= \begin{cases}I_{e 0} \exp \left(\frac{e \phi}{k_{B} T_{e}}\right) & (\phi<0 \mathrm{~V}) \\ I_{e 0}\left(1+\frac{e \phi}{k_{B} T_{e}}\right)^{\alpha} & (\phi \geq 0 \mathrm{~V}),\end{cases}
$$

where

$$
I_{e 0}=\mu A_{S} n_{0} e \sqrt{\frac{k_{B} T_{e}}{2 \pi m_{e}}} .
$$

$A_{S}$ is the total surface area of the spacecraft, $T_{e}$ is an ambient electron temperature, $m_{e}$ is the mass of an electron, $n_{\mathrm{e}}$ is an ambient electron density. The coefficient $\mu$ is 1.0 for a spherical object, and $\frac{2}{\sqrt{\pi}} \sim 1.1$ for an infinite cylinder. The coefficient $\mu$ did not included the original form of the OML theory by [18]. $\mu$ was first derived by Laframboise [19] and the expressions here is by Lai [21]. In the computations in this paper, we used $\mu=1.0$ for all cases since there is no significant difference in the charge potential. The power factor $\alpha$ is 1 if the object is a perfect sphere, $\alpha=1 / 2$ if it is an infinite cylinder and 0 if it is an infinite plate [17] (here "infinite" can be seen as large when compared to a typical length).

Ion currents can also be described by the OML theory. The ambient proton $\left(\mathrm{H}^{+}\right)$current for the Maxwellian distribution is

$$
I_{\mathrm{H}^{+}}(\phi)= \begin{cases}I_{\mathrm{H}_{0}^{+}} \exp \left(-\frac{e \phi}{k_{B} T_{i}}\right) & (\phi \geq 0 \mathrm{~V}) \\ I_{\mathrm{H}_{0}^{+}}\left(1-\frac{e \phi}{k_{B} T_{i}}\right)^{\alpha} & (\phi<0 \mathrm{~V}),\end{cases}
$$

where

$$
I_{\mathrm{H}_{0}^{+}}=\mu A_{S} n_{\mathrm{H}^{+}} e \sqrt{\frac{k_{B} T_{i}}{2 \pi m_{i}}}
$$

$T_{i}$ is an ambient ion temperature, $n_{\mathrm{H}^{+}}$is a proton density, and $m_{\mathrm{H}}$ is the mass of a proton. When accounting for multiple species, there are as many terms in the total ion current as species considered, each with their respective charge and atomic mass. Here our computation model includes $\mathrm{H}^{+}, \mathrm{He}^{+}$, $\mathrm{O}^{+}, \mathrm{O}^{2+}$ ions. The ambient ion temperature $T_{i}$ is the same value for all ion species.

Additionally, in LEO, ion currents contribute as a drift current induced by the spacecraft velocity 
$V_{\mathrm{SC}}$ with respect to the plasma. For instance, the drift current by proton can be written as

$$
I_{\mathrm{H}^{+} \mathrm{drift}}=A_{S} n_{\mathrm{H}^{+}} e V_{s c}
$$

Thus, the total inflow current by ions becomes

$$
\begin{aligned}
I_{i} & =I_{\mathrm{H}^{+}}+I_{\mathrm{H}^{+} \text {drift }}+I_{\mathrm{He}^{+}}+I_{\mathrm{He}^{+} \text {drift }} \\
& +I_{\mathrm{O}^{+}}+I_{\mathrm{O}^{+} \text {drift }}+I_{\mathrm{O}^{2+}}+I_{\mathrm{O}^{2+} \text { drift }} .
\end{aligned}
$$

Note that the OML theory is the plasma collection theory for a small object compared to Debye length, which is the representative length of the ambient plasma (detailed definitions are given in III A 2). When the ratio between the representative size of the object and the Debye length becomes large $(>1)$, the power factor $\alpha$ will decrease and finally $\alpha \rightarrow 0$, because the effective collection area becomes smaller. In LEO, Debye length is quite small (several centimeter to millimeter) so that the exact current collection in LEO will have smaller $\alpha$ than in GEO, particularly for large objects. However, currently $\alpha$ can only be obtained from the experimental result [21] and there is no exact method to estimate $\alpha$ from the ratio between the representative size of the object and Debye length. Since the current collection in LEO has smaller $\alpha$, namely, is less sensitive to the object's potential, the magnitude of the object's potential given by the OML theory will describe the minimum (absolute) potential in LEO. Hence, we employed the OML theory in this paper even though we focus on the charging of relatively large object in LEO.

b. Photoelectron current The photoelectron current $I_{p h}$ is defined as:

$$
I_{p h}=s A_{p} J_{p h 0}
$$

where $J_{p h 0}=3 \times 10^{-5} \mathrm{~A} / \mathrm{m}^{-2}$ is the current density according to [22] and $A_{p}$ the projected area with respect to the flow of photons. For a spherical object of radius $R, A_{p}=\pi R^{2}=A_{S} / 4$. Recall that $s$ is the shadow function.

Since the electron flux is bigger than the ion flux in LEO, $\phi$ is usually negative in eclipse. However, in sunshine, the photoelectron current basically renders the potential less negative, and can even make it positive when the photoelectron exceeds the ambient electron current. 


\section{Electrostatic capacitance}

In space, the charged environment affects the capacitance $C$. The ambient plasma is attracted by the potential of an object and forms a sheath structure around it. A representative length of the sheath $\lambda_{D}$ - the so-called Debye length - is defined as [23]:

$$
\begin{aligned}
& \lambda_{D e}=\sqrt{\frac{\varepsilon_{0} k_{B} T_{e}}{n_{e} e^{2}}} \quad \text { (for electron), } \\
& \lambda_{D i}=\sqrt{\frac{\varepsilon_{0} k_{B} T_{i}}{n_{i} e^{2}}} \quad \text { (for ion), } \\
& \frac{1}{\lambda_{D}^{2}}=\frac{1}{\lambda_{D e}^{2}}+\frac{1}{\lambda_{D i}^{2}} .
\end{aligned}
$$

The final expression of the capacitance itself depends on the geometry of the object. The case of a sphere and a flat plate are given thereafter. Note that, in what follows, the vacuum permittivity $\varepsilon_{0}$, representing a perfect conductor, is used rather than an absolute one $\varepsilon>\varepsilon_{0}$, characterizing a dielectric surface. The reason for this is that the former can induce partial charging, which is fairly complex to model.

a. Sphere The formula for the capacitance between two spherical conductors with respective radius $a$ and $b$ is classically known as:

$$
C_{\text {sphere }}=\frac{4 \pi \varepsilon_{0} R}{1 / a-1 / b}
$$

where $b>a$. Assuming that the total interacting plasma is contained between two concentric conductive spheres [8], one has $a=R$ and $b=R+\lambda_{D}$. Thus it comes that:

$$
C=\frac{4 \pi \varepsilon_{0} R\left(R+\lambda_{D}\right)}{\lambda_{D}}
$$

Since $\lambda_{D}$ is usually on the order of centimeter to millimeter in LEO, for a large object, one has $R / \lambda_{D} \gg 1$ and thus Eq.(23) becomes:

$$
C=\frac{4 \pi \varepsilon_{0} R^{2}}{\lambda_{D}}=\frac{4 \varepsilon_{0} A}{\lambda_{D}}
$$

where $A$ is the cross-sectional area. Space debris smaller than $1 \mathrm{~cm}$ do not satisfy this condition and for them Eq.(23) should be used. Nonetheless, when Eq.(24) is valid then one can see that the acceleration due to the magnetic field is proportional to the area-to-mass ratio $A / m$ because the 
acceleration by Lorentz force writes:

$$
\begin{aligned}
\ddot{\mathbf{r}}_{L} & =\frac{q}{m} \mathbf{v} \times \mathbf{B}, \\
& =\frac{C \phi}{m} \mathbf{v} \times \mathbf{B}, \\
& =\frac{A}{m} \frac{4 \varepsilon_{0} \phi}{\lambda_{D}} \mathbf{v} \times \mathbf{B} .
\end{aligned}
$$

About the effect of plasma sheath, note that there is no consensus on the fact that all the particles in the Debye sphere contribute to the Lorentz force. If it is true, Eq.(23) and Eq.(24) are perfectly valid. If it is not true, the realistic capacitance (and thus the charge) is somewhat smaller than Eq.(23).

b. Plate For two parallel plates separated by a distance $d$, the capacitance be written as:

$$
C_{\text {plate }}=\frac{\varepsilon_{0} S}{d}
$$

where $S$ is the surface area of each plate. Assuming that the plasma interacts with the surface of the object up to a distance equal to the Debye length, one can estimate the capacitance $C$ as:

$$
C=\frac{\varepsilon_{0} A}{2 \lambda_{D}}
$$

where the factor $1 / 2$ comes from the fact that both sides of the object are charged (the total capacitance of two similar capacitance in series is half of one.) It is worth noticing that, also for the plate, the Lorentz force is linear with respect to the area-to-mass ratio.

\section{Ionosphere}

In LEO, objects evolve in the ionosphere, as it spans roughly from $100 \mathrm{~km}$ to $3000 \mathrm{~km}$. It is the part of the upper atmosphere where a significant amount of charged particles can be encountered due to solar radiation. The ionosphere is structured in layers where physical quantities e.g. species densities follow similar trends. There is a clear dichotomy between the day-time and night-time sides, as well as latitudinal and seasonal variations. The ionospheric model used here is based on the so-called International Reference Ionosphere (IRI) [24]. A brief description of its content is given thereafter based on the editions of 1990 and 2012, even though only the latter has been used here to run simulations. 
a. Plasma temperature Electron temperature $T_{e}$ is crucial for the magnetic force because it drives the electron current. In IRI-1990, $T_{e}$ is described via a so-called Booker profile [25]. It basically consists in a piece-wise affine function with smooth transitions between successive layers. The boundaries are points from various references that have a dependency on local time and latitude as well as seasons for some of them.

Similarly to electron temperature, ion temperature influences spacecraft charging, but to a lower extend. According to IRI-1990, ion temperature $T_{i}$ equals electron temperature over $1000 \mathrm{~km}$ and is always smaller at lower altitudes.

b. Plasma densities Electron and ion densities mainly play a role in the computation of the Debye length $\lambda_{D}$ for the electrostatic capacitance and thus influence the value of the charge $q$. As a plasma is globally neutral, one has $n_{e}=n_{i}$. The IRI model also gives the ratios for the different species among the ions $\left(\mathrm{O}^{+}, \mathrm{H}^{+}, \mathrm{He}^{+}\right)$.

\section{B. In MEO}

Unlike the IRI-2012 in LEO, there is no standard plasma environment model for MEO at present to the best of the authors' knowledge. In this paper, a very simple extremal model is used in this region, based on the surface potential observed by Van Allen Probes [26]. According to this reference, the surface potential in eclipse is approximately one hundred percent negative (-10V to 0V). Also, in sunshine, the potential becomes 0V to 10V. Here, above $2000 \mathrm{~km}$ which is the maximum altitude of IRI-2012, it is considered that

$$
\phi=-5+10 s \mathrm{~V}
$$

where $s$ is the shadow function previously introduced.

As for the electrostatic capacitance, the formulas remain unchanged with respect to the LEO case. The only needed local quantity is the Debye length, with is fixed a priori to $10 \mathrm{~m}$.

\section{Results}

The model described in Section II has been run to analyze the effects of the magnetic force on space trajectories. More precisely, the idea is to see how the Keplerian coordinates of a charged 
object evolve with respect to those of an electrically neutral one, starting from the same initial conditions.

In all simulations, the area-to-mass ratio is fixed to $10 \mathrm{~m}^{2} / \mathrm{kg}$ in order to maximize the effects of non-gravitational perturbations, but even bigger values can be found in the literature [3, 4]. Two initial inclinations are simulated: 89.0 and 0.1 degrees. In the polar configuration, the object experiences all magnetic latitudes within one orbital period and all longitudes in the span of several days, while for the equatorial case, the debris sees the full range of magnetic longitudes within an orbit and encounters daily eclipses. The initial eccentricity, right ascension of the ascending node $\Omega$, argument of the perigee $\omega$ and true anomaly are listed in Table 1. The initial semi-major axis $a$ depends on the regime considered: here $8000 \mathrm{~km}$ for the case in LEO and $20000 \mathrm{~km}$ for MEO. Investigating long-term effects prevents from using an initial altitude that is too low, otherwise the orbit decay would be so strong that it would mask all other perturbations and cause re-entry very quickly. The choice of the other initial Keplerian coordinates (eccentricity, right ascension of the ascending node, argument of perigee and true anomaly) being arbitrary, they were set to match the LEO case in [8]. In particular, the orbit is initially quasi-circular.

All the parameters related to the orbital perturbations are given in Table 2, including the mean solar flux $F_{10.7}$ and the geomagnetic index $K_{p}$ when applicable. The latter are kept constant during the propagation, assumption that can be considered as unreasonable over a long timespan, but it is to be remembered that the topic of interest here is the individual and coupled effects of the magnetic force, not the aerodynamics itself, so that a high accuracy is not necessary for the latter. As far as gravity is concerned, the geopotential is expanded with coefficients from EGM96 up to order and degree 29 in LEO and 14 in MEO. As for the magnetic potential of the Earth, the expansion is done to the 8th order and degree (from IGRF-12), including secular variations of the Gauss coefficients. The initial epoch is $21 / 3 / 2000$ at 12:00 UTC, as the use of IRI12 is limited to the period before 2019 .

\section{A. LEO simulations}

Two geometries are investigated in LEO: a sphere and a square plate. The first one is simulated using a 3dof propagator while the second one requires a 6dof model. Integration step-size is set to 
Table 1: Initial orbital elements

\begin{tabular}{|c|c|c|c|}
\hline eccentricity (-) & $\Omega(\mathrm{deg})$ & $\omega(\mathrm{deg})$ & true anomaly $(\mathrm{deg})$ \\
\hline 0.001 & 60 & 30 & 100 \\
\hline
\end{tabular}

Table 2: Other parameters

\begin{tabular}{|c|c|c|c|c|}
\hline $\mathrm{A}\left(\mathrm{m}^{2}\right)$ & $\mathrm{m}(\mathrm{kg})$ & $C_{R}(-)$ & $F_{10.7}\left(\mathrm{~W} / \mathrm{s}^{2}\right)$ & $K_{p}(-)$ \\
\hline 1 & 0.1 & 1.3 & $187 \times 10^{-22}$ & 1 \\
\hline
\end{tabular}

$30 \mathrm{~s}$ for the former and to $0.5 \mathrm{~s}$ for the latter. Orbital state and other quantities of interest are saved every 2 hours.

\section{Sphere in $L E O$}

Two subcases are considered, in order to investigate interactions between accelerations in the model: with and without taking into account the solar flux. Recall that, in summary, the solar flux produces a photoelectron surface current, contributing to the Lorentz force, as well as a radiation pressure that further perturbs the object motion.

a. Polar orbit Figure 1 shows both the surface potential and electrostatic charge versus time for the polar orbit, i.e. $\mathrm{i}=89$ deg. In order to visualize the orbit decay, Figure 2 depicts the osculating semi-major axis versus time for the non-charged object. Figures 3 to 6 show the differences between the Keplerian coordinates propagated with and without surface charging for a sphere on a polar orbit with and without the solar flux.

The potential is of the order of one Volt whereas the absolute value of the surface charge is of the order of a few nano Coulomb. The main difference between the two cases (with and without solar flux) comes from the fact that different local plasma-related conditions are encountered on the orbit as the same initial orbital elements evolve differently with or without the solar radiation pressure. In particular, the latter excites the eccentricity, lowering the altitude of the perigee and thus causing a faster orbit decay. Additionally, it is noticeable that the photoelectron current tends to shift the surface potential in the positive direction, leading to some positive values of the electrostatic charge, 

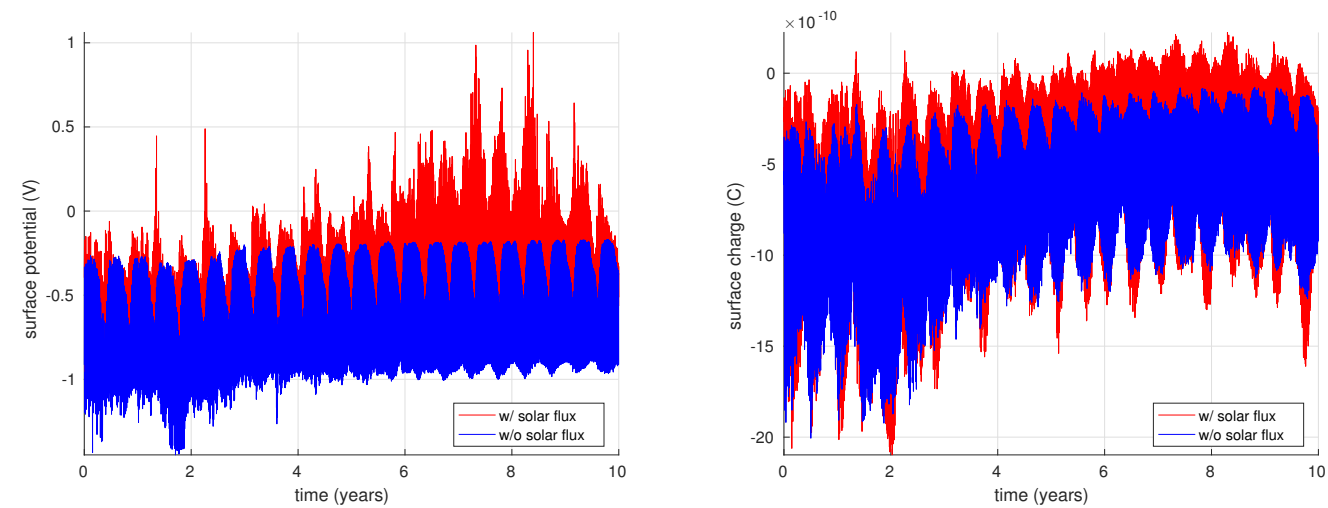

Fig. 1: Time history of surface potential (left) and electrostatic charge (right) in polar LEO (3dof)

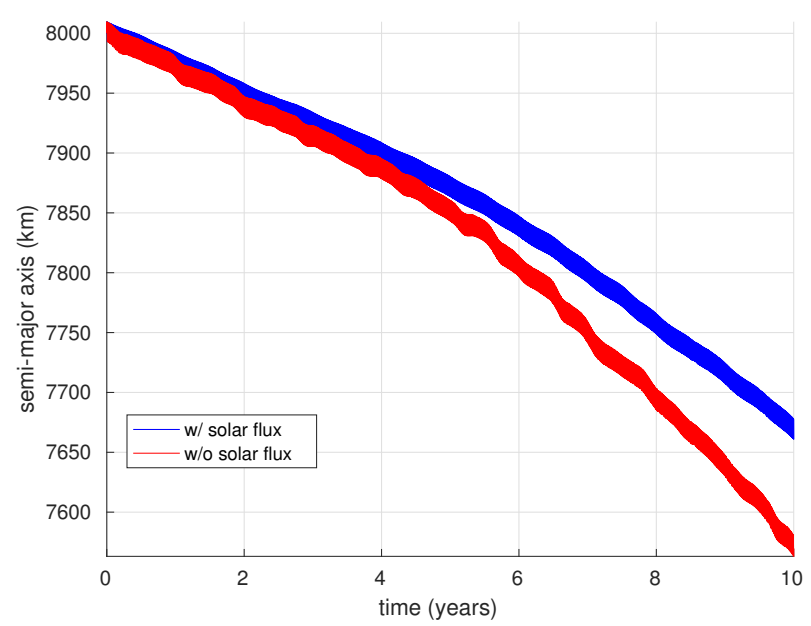

Fig. 2: Time history of osculating semi-major axis for the non-charged object in polar LEO (3dof)

which never happens in the other case where the photoelectron current is not included in the balance equation.

Without the solar flux, the impact of the Lorentz force over the course of ten years is rather small, to the point where differences blend with integration errors. The orders of magnitude are $10^{-1} \mathrm{~km}$ for the semi-major axis, $10^{-5}$ for the eccentricity, $10^{-5}$ degrees for the inclination and $10^{-3}$ for the right ascension of the ascending node. Note that the argument of perigee is not shown as it without the solar flux the orbit remains quasi-circular and thus this parameter does not make much sense in that case.

Differences in Keplerian coordinates tend to grow over time, but vanish on average for the parameters describing the shape of the orbit (semi-major axis and eccentricity). On the contrary, the average 

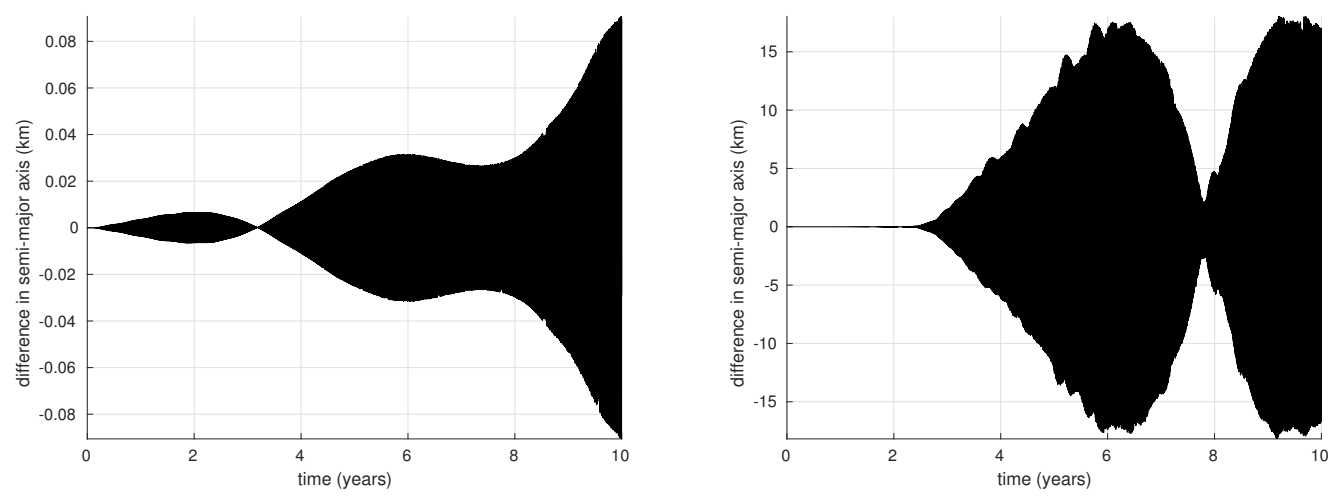

Fig. 3: Time history of difference in osculating semi-major axis without (left) and with (right) the solar flux in polar LEO (3dof)
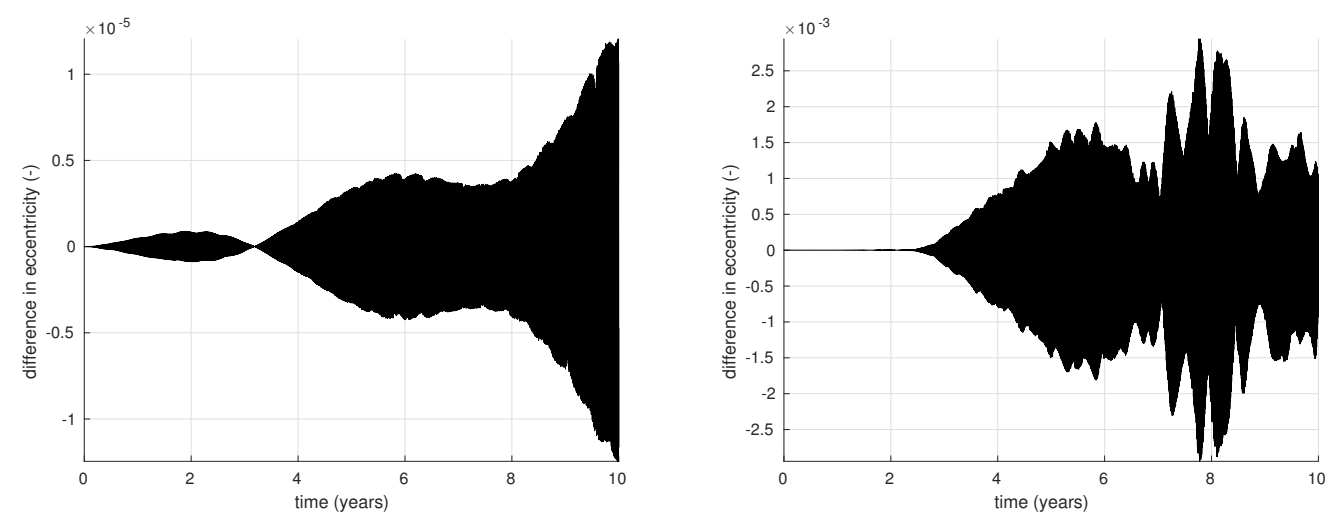

Fig. 4: Time history of difference in osculating eccentricity without (left) and with (right) the solar flux in polar LEO (3dof)

is non-zero for the elements related to the plane of the orbit. In particular, there is an almost linear long-term evolution for $\Omega$ that can be explained by the fact that the magnetic force partly acts perpendicular to the orbital plane. As a result, it adds a steady contribution to its rotation rate, which is fully transmitted to the right ascension of the ascending node in a polar configuration.

With the solar flux, the differences in Keplerian coordinates are one to two orders of magnitude larger than without. This can be explained by the coupling with eclipses, when the radiation pressure vanishes. As the magnetic force slowly creates a shift in position between charged and non-charged objects, they start being in eclipse conditions at slightly different times, which in turn excites this divergence in the trajectory due to the difference in forces acting on the object. It is to be noted 

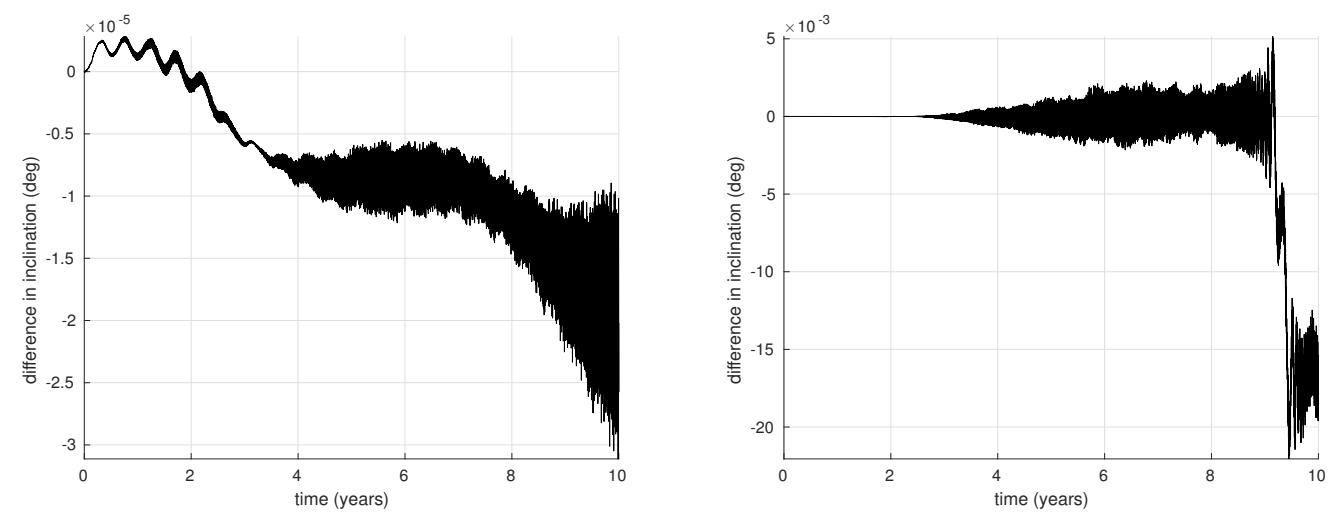

Fig. 5: Time history of difference in osculating inclination without (left) and with (right) the solar flux in polar LEO (3dof)
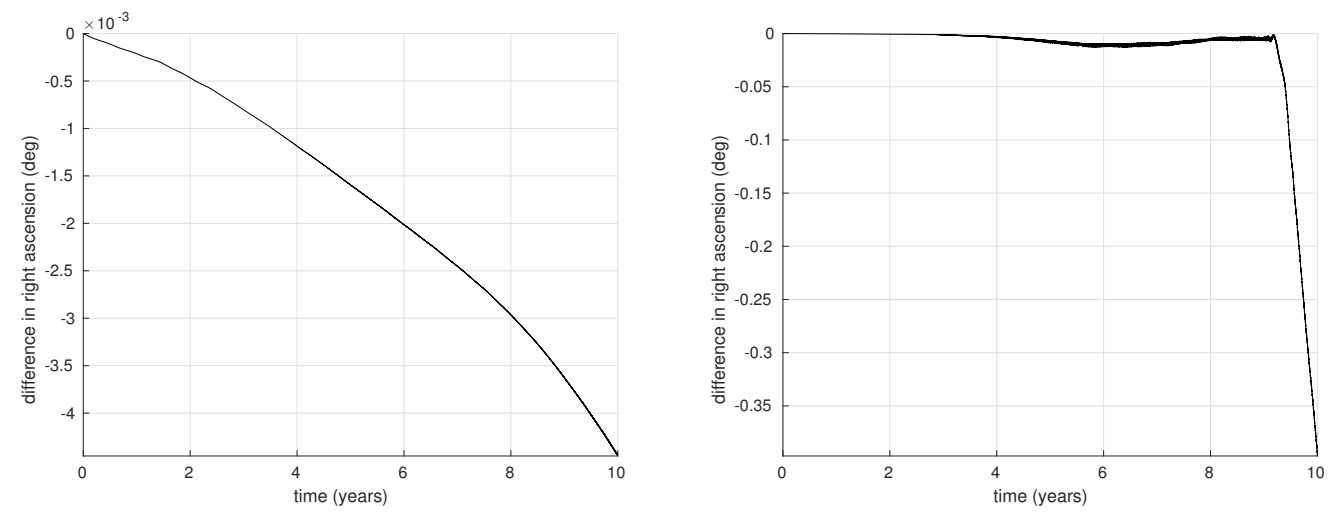

Fig. 6: Time history of difference in osculating right ascension of the ascending node without (left) and with (right) the solar flux in polar LEO

that the numerical translation of that phenomenon is highly dependent on the expression of the shadow function. Results previously obtained by the authors in [9] were more pronounced due to a larger step-size in the integration as well as a different model i.e. a sigmoid between penumbra and umbra. It is worth noticing that the one currently used, based on the degree of Sun's occultation, does not have continuous high-order derivatives, which violates the usual assumption of numerical integrators. On another note, the trend in inclination and right ascension of the ascending node seems to change towards the end of the simulation, around year 10. This depends on the natural evolution of the orbit under the solar radiation pressure (generating high eccentricity, while the geopotential makes it drift), as well as the positive values of surface charge appearing at this date. 
b. Equatorial orbit Figure 8 shows surface potential and electrostatic charge as functions of time for equatorial orbit. The curves are similar to the polar case. Figure 8 depicts the evolution of the osculating semi-major axis of the non-charged object. It demonstrates closer decay rates than previously seen, due to large portions on the orbit being in umbra, where the solar radiation pressure vanishes. Figures 9 to 11 depict the differences between the Keplerian coordinates propagated with and without surface charging. The right ascension of the ascending node is not shown as it is not defined for equatorial orbits. Orders of magnitude are very similar to the polar case.
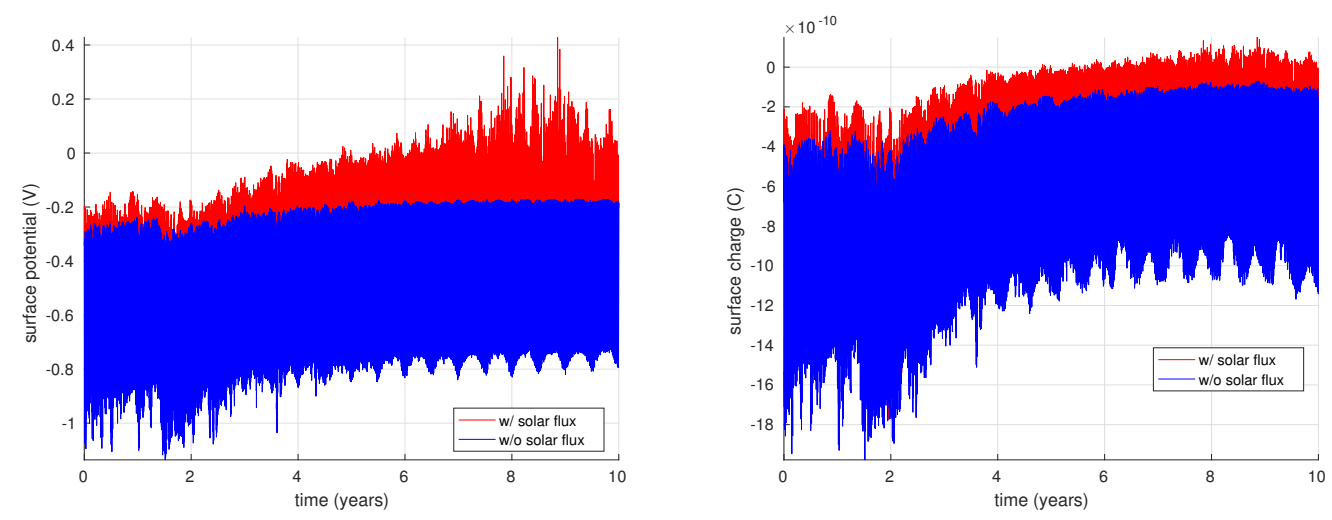

Fig. 7: Time history of surface potential (left) and electrostatic charge (right) in equatorial LEO (3dof)

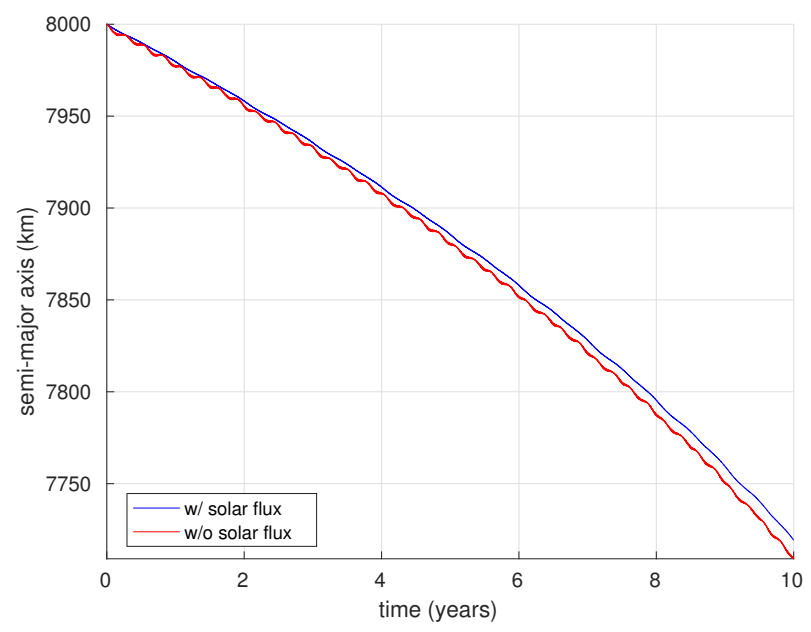

Fig. 8: Time history of osculating semi-major axis for the non-charged object in equatorial LEO (3dof) 

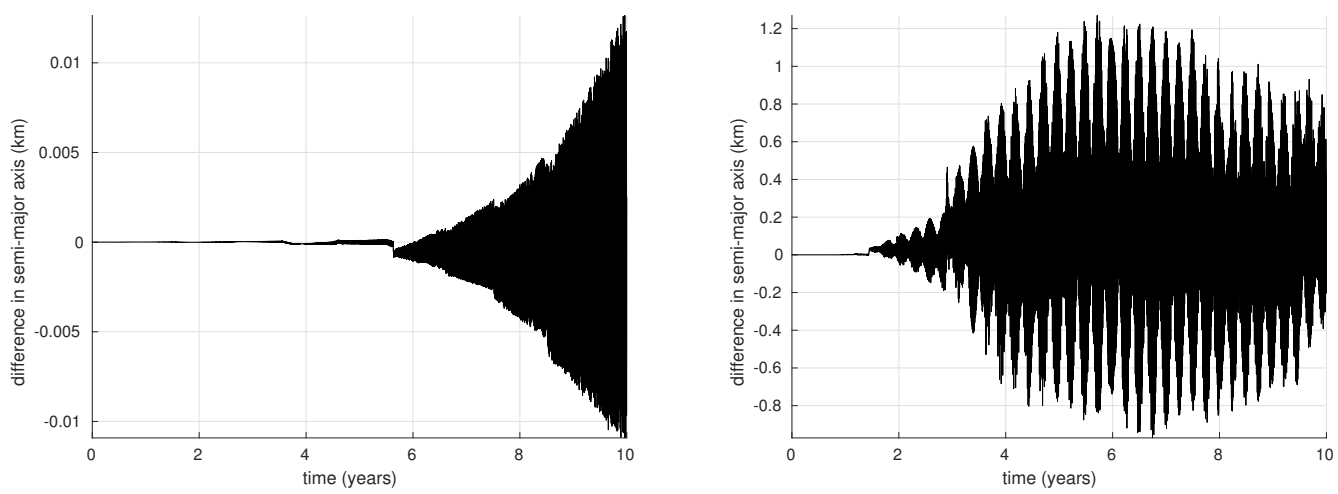

Fig. 9: Time history of difference in osculating semi-major axis without (left) and with (right) the solar flux in polar LEO (3dof)
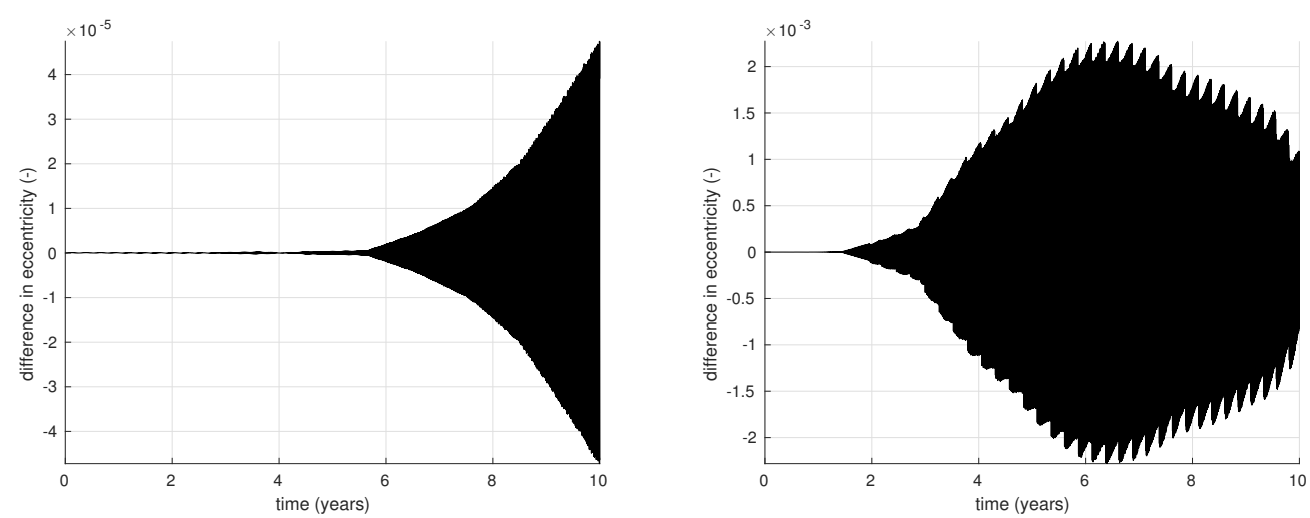

Fig. 10: Time history of difference in osculating eccentricity without (left) and with (right) the solar flux in polar LEO (3dof)

\section{Square plate in LEO}

Studying a non-spherical object allows for a study of the effects of surface charging on attitude. 6dof analysis is also required to justify the viability of the cannonball assumption for drag. In order for the Lorentz force to induce a torque on the barycenter, a displacement of the latter with respect to the center of symmetry is introduced. It is achieved by considering a square plate made of two half plates of similar, rectangular dimensions, but with different, uniform mass distributions. Let $(y, z)$ be the plane of the whole plate and $x$ the orthogonal direction. Let $l$ be the total length of the square plate and $w$ its width while $m_{1}, m_{2}$ denote the mass of each half plate (with dimensions $l \times l / 2 \times w)$. The overall geometry of the object is shown in Figure 12. The principal moments of 

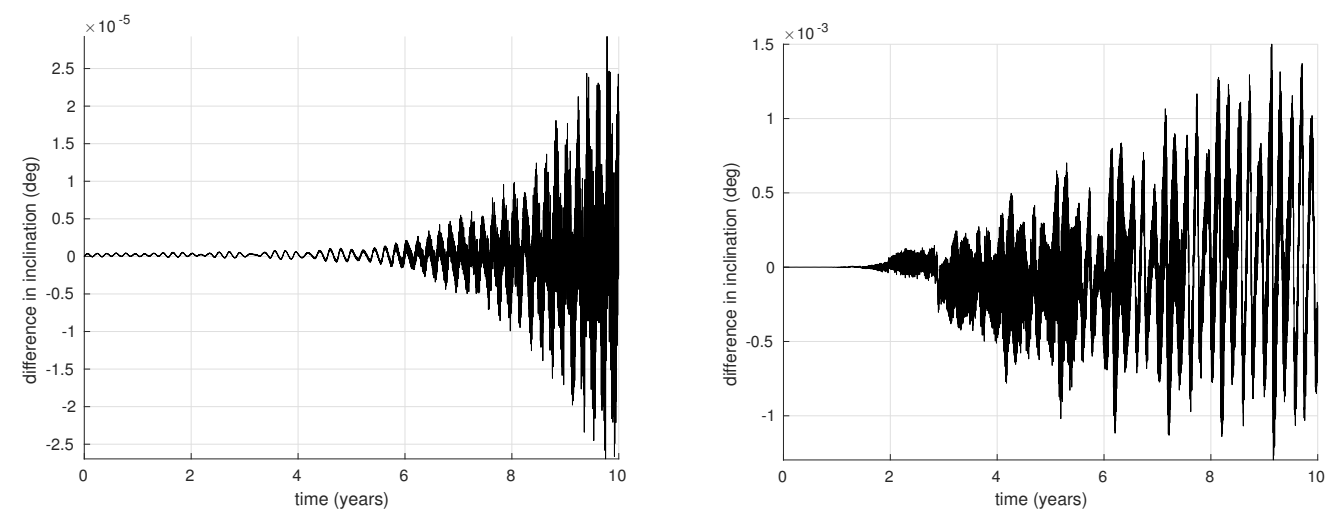

Fig. 11: Time history of difference in osculating inclination without (left) and with (right) the solar flux in polar LEO (3dof)

inertia of the objects around the barycenter can be obtained via the parallel axis theorem. If the junction between the two rectangular plates is parallel to the $z$-direction, then they write:

$$
\begin{aligned}
& J_{x}=\frac{l^{2}\left(m_{1}+m_{2}\right)}{4}\left(\frac{5}{12}+\frac{m_{1}}{m_{1}+m_{2}}\left(1-\frac{m_{1}}{m_{1}+m_{2}}\right)\right) \\
& J_{y}=\frac{\left(m_{1}+m_{2}\right)}{12}\left(l^{2}+w^{2}\right) \\
& J_{z}=\frac{\left(m_{1}+m_{2}\right)}{12}\left(\frac{l^{2}}{4}+w^{2}+\frac{3 l^{2} m_{1}}{m_{1}+m_{2}}\left(1-\frac{m_{1}}{m_{1}+m_{2}}\right)\right)
\end{aligned}
$$

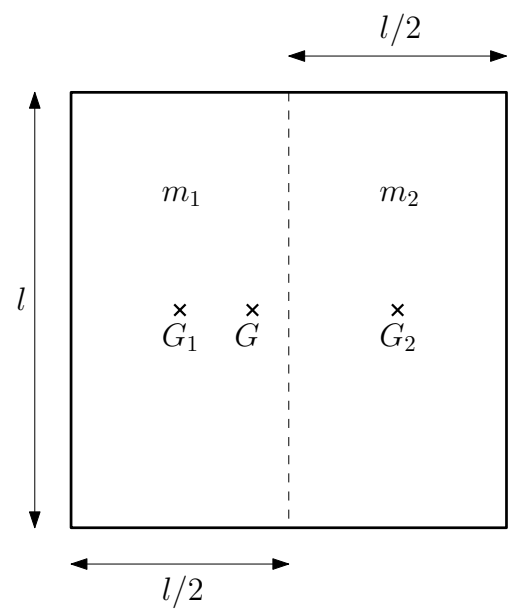

Fig. 12: Upside view of the square plate with non-homogeneous mass distribution

The total length $l$ is set to $1 \mathrm{~m}$ and the total mass $m_{1}+m_{2}$ to $0.1 \mathrm{~kg}$. Note that the values of these two quantities are not very important as long as the area-to-mass ratio is still 10. Initial 
conditions for attitude are given in Tables 3 and 4 . In the following 6dof simulations, the solar flux is not taken into account, meaning that radiation pressure and photoelectron current are not included in the model. This allows to focus on the direct, non-coupled effects of the magnetic torque.

Table 3: Initial quaternions

\begin{tabular}{|c|c|c|c|}
\hline$Q_{1}(-)$ & $Q_{2}(-)$ & $Q_{3}(-)$ & $Q_{4}(-)$ \\
\hline 0.0 & 0.365998 & 0.211309 & 0.642788 \\
\hline
\end{tabular}

Table 4: Initial angular rates

\begin{tabular}{|c|c|c|}
\hline$\omega_{1}(\mathrm{rad} / \mathrm{s})$ & $\omega_{2}(\mathrm{rad} / \mathrm{s})$ & $\omega_{3}(\mathrm{rad} / \mathrm{s})$ \\
\hline 0.01 & -0.02 & 0.03 \\
\hline
\end{tabular}

The following 6dof simulations are only 0.1 year long and restricted to the polar orbit, due to their greater computational cost. Figure 13 shows the surface potential and charge as functions of time. Figures 14 and 15 depict the differences in osculating orbital elements.
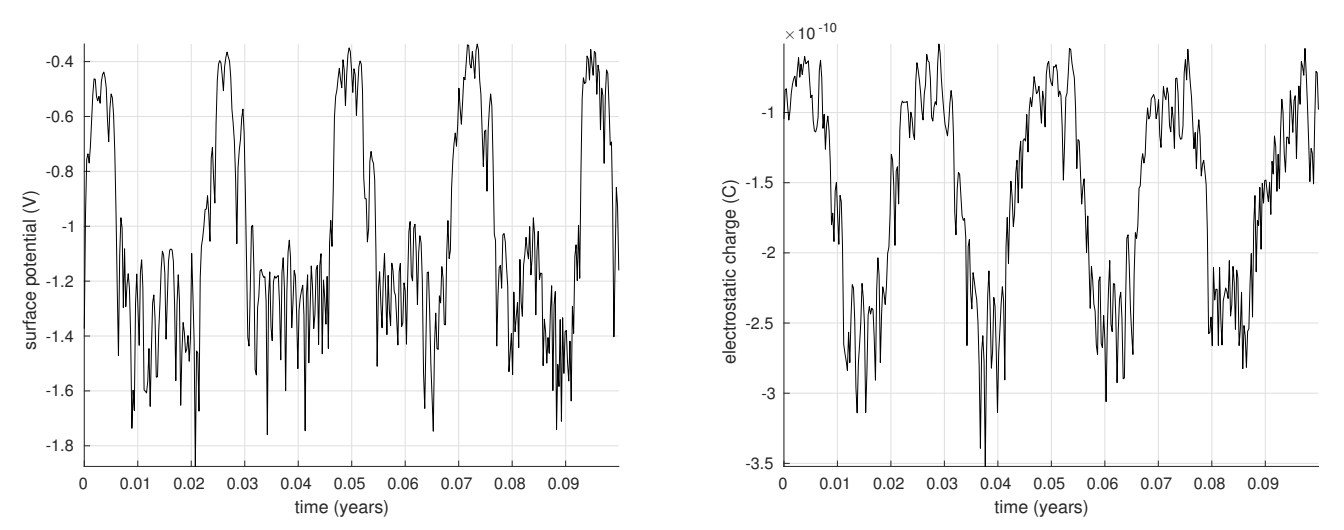

Fig. 13: Time history of surface potential (left) and electrostatic charge (right) in polar LEO

Similarly to the spherical geometry, the surface potential has a magnitude around 1V. As for the surface charge, it is around $0.1 \mathrm{nC}$. This drop of about one order of magnitude is due to the difference in the charging model of the two geometries. In particular, the electrostatic capacitance differs by a factor 8 (see Eq.(23) and (29)). This reduction partly translates into the differences 

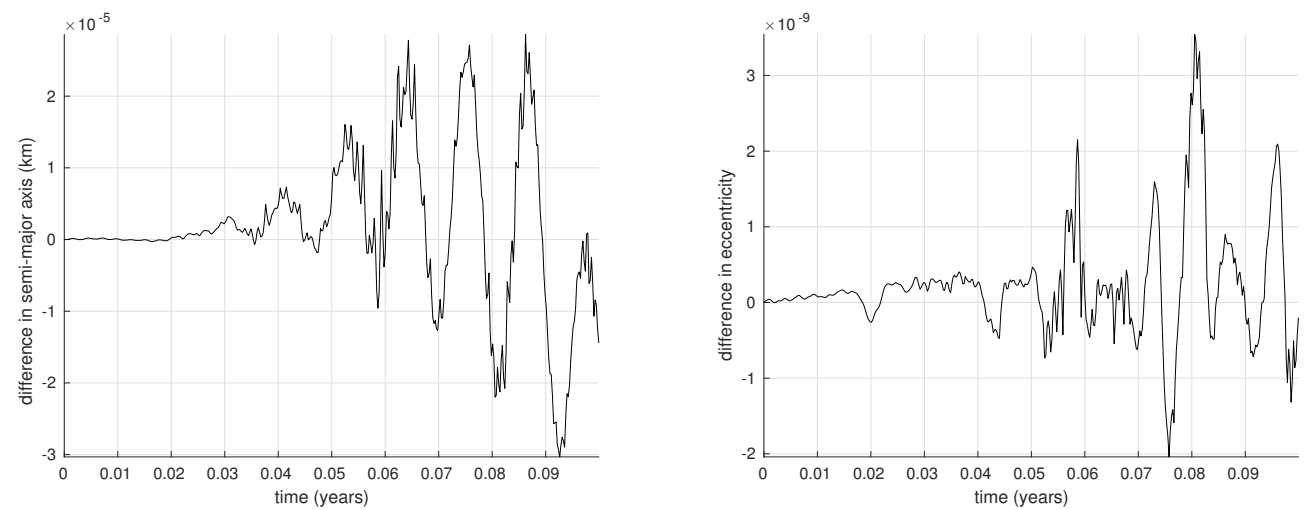

Fig. 14: Time history of difference in osculating semi-major axis (left) and eccentricity (right) in polar LEO (6dof)
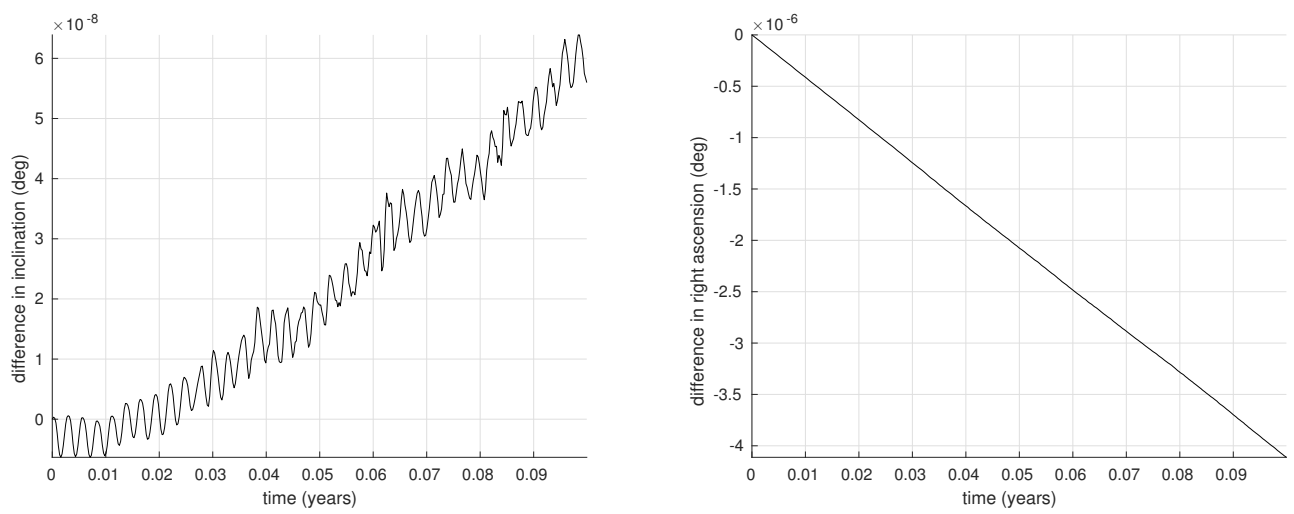

Fig. 15: Time history of difference in osculating inclination (left) and right ascension of the ascending node (right) in polar LEO (6dof)

in orbital elements that are generally smaller than for the sphere after one year in the same case (without solar flux).

The interesting result from the plate is that the attitude evolves in a very different way between the charged and the non-charged case. This phenomenon can be seen in Figure 16, which shows the time history of differences in quaternions. After a few weeks, the discrepancy is already of the order of 0.1. It is non-negligible as these quantities are always between -1 and 1 . This mean that the torque induced by the surface charge and the non-homogeneous mass distribution is large enough to significantly change the history of attitude over time. On the other hand, it is also worth mentioning that the object simulated is so light that its attitude motion has a somehow chaotic behavior, in the 
sense that a slight change in the initial conditions or any other parameter can have a huge impact on the evolution of the system. However, comparing charged and non-charged objects without a displacement of the barycenter (and hence no torque apart from the gravity gradient) demonstrates a negligible difference in attitude dynamics i.e. of the order of $10^{-7}$ for each quaternion.

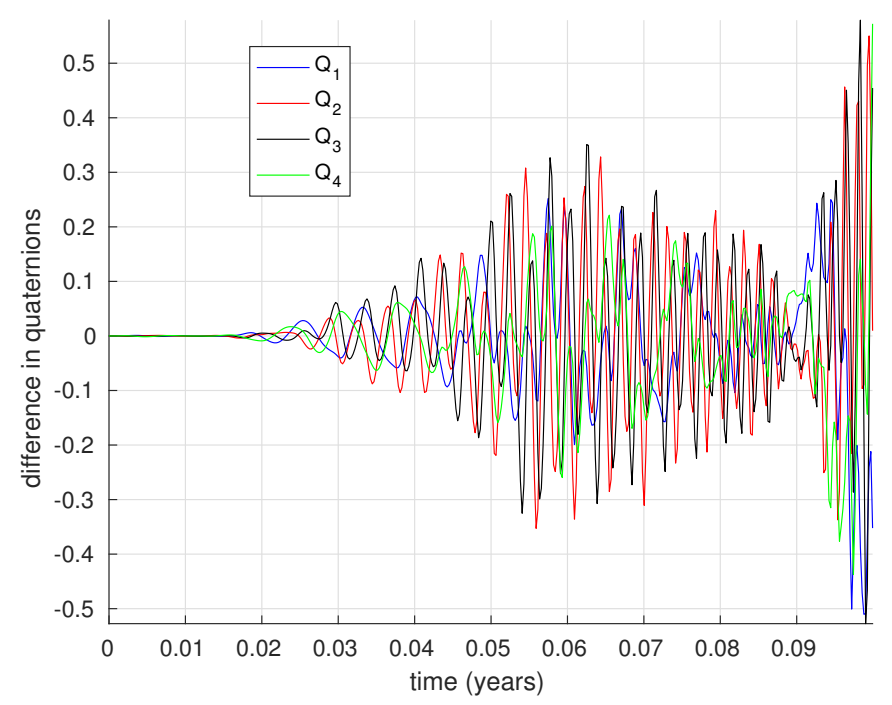

Fig. 16: Time history of differences in quaternions

\section{B. MEO simulations}

Since the adopted charging model highly depends on eclipse conditions, the solar radiation pressure is accounted for in the MEO simulation. At these altitudes, atmospheric drag is nonexistent, so that there is no orbit decay, allowing for longer integration. Simulations are limited to 3dof as light pressure introduces a chaotic behavior in the attitude dynamics of objects with a high area-to-mass ratio. Figures 17 to 18 depict the time history over 100 years of differences in Keplerian coordinates between charged and non-charged spherical objects in a polar configuration $(i=89 \mathrm{deg})$. Unlike the LEO simulations, the frequency for recording data is 24 hours.

Orders of magnitude for the difference in mean orbital elements are the following after a century: $10^{-1} \mathrm{~km}$ for the semi-major axis, $10^{-5}$ for the eccentricity, $10^{-5}$ degrees for the inclination and $10^{-4}$ for the right ascension of the ascending node. After 10 years, these numbers are much smaller than the LEO case, and several reasons can explain that. On one hand, the magnitude of the cross 
product between the magnetic field and the spacecraft velocity decreases rapidly with altitude. In fact, assuming a dipole model and an orbital speed equal to the circular one, $\|\mathbf{v} \times \mathbf{B}\|$ scales as $r^{-7 / 2}$. On the other hand, the surface charge is smaller as it is inversely proportional to the Debye length which is about 3 orders of magnitude higher in MEO than in LEO.
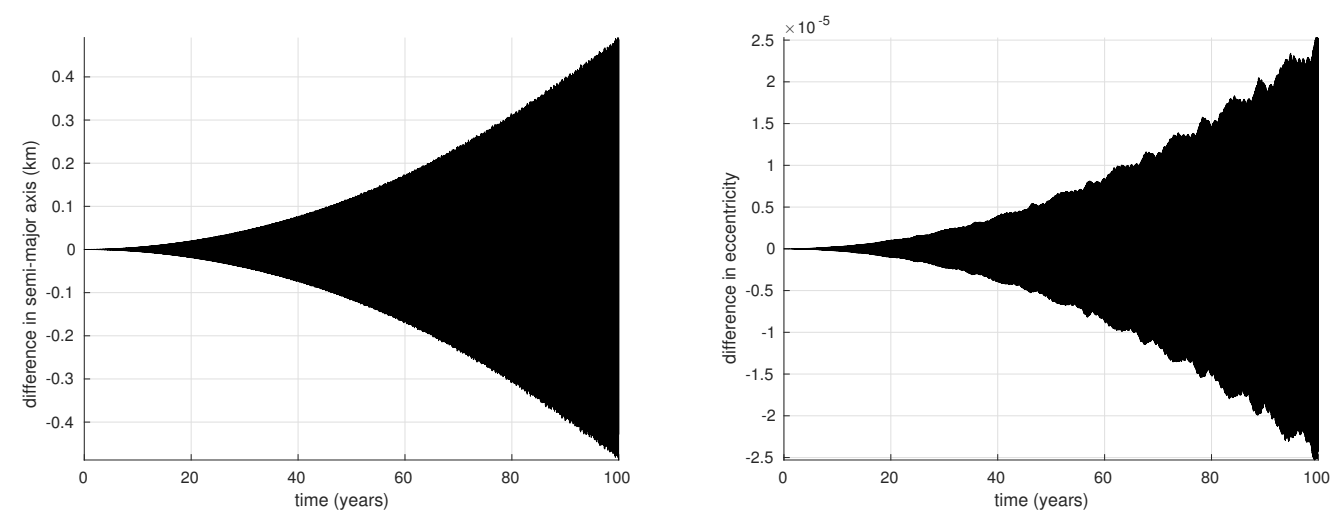

Fig. 17: Time history of difference in osculating semi-major axis (left) and eccentricity (right) in polar MEO (3dof)
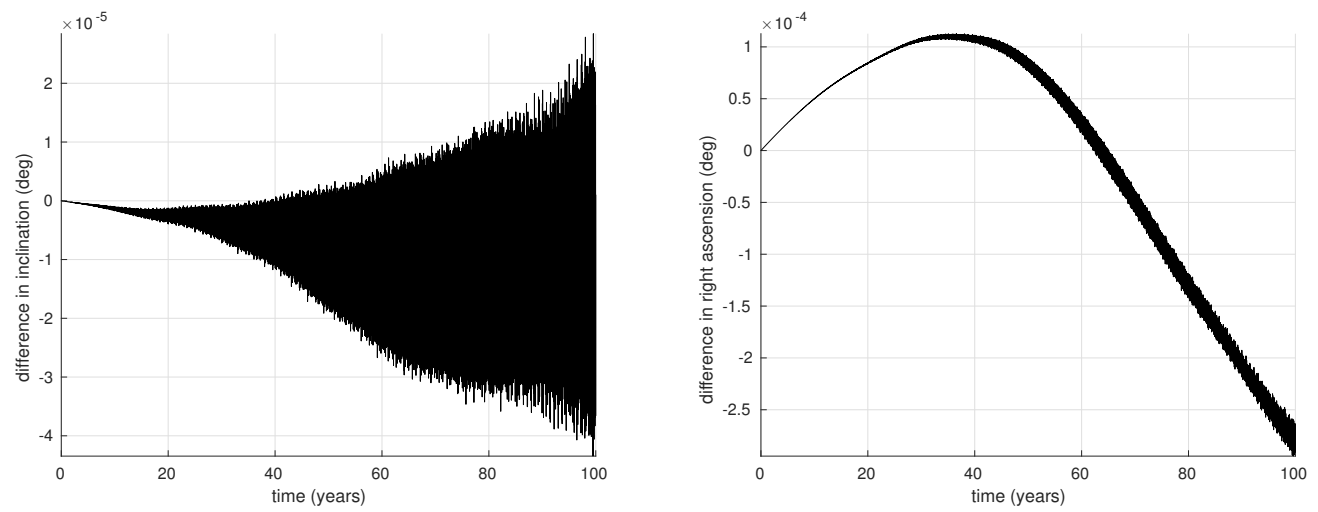

Fig. 18: Time history of difference in osculating inclination (left) and right ascension of the ascending node (right) in polar MEO (3dof)

In order to put things into perspectives, the same MEO model has been applied to dust particles. More precisely, a grain was considered, with radius $1 \mu \mathrm{m}$ and mass $14.188790204786391 \times 10^{-15} \mathrm{~kg}$, corresponding to an area-to-mass ratio of 750 i.e. two orders of magnitude above the previous space debris. This represents another range of values describing a very difference type of space objects for which orbital perturbations such as solar radiation pressure almost become dominant over gravity. 
Figures 19 to 20 show the history of Keplerian coordinates for two particles starting from the same initial conditions as before, but only one is charged while the other is not. The comparison stops when the non-charged grain reenters. It is noticeable that the radiation pressure is so important that the eccentricity oscillates widely, causing atmospheric re-entry within a year. As one can see, the Lorentz force plays a major part in the dynamics, as the two trajectories start diverging significantly on the scale of days.
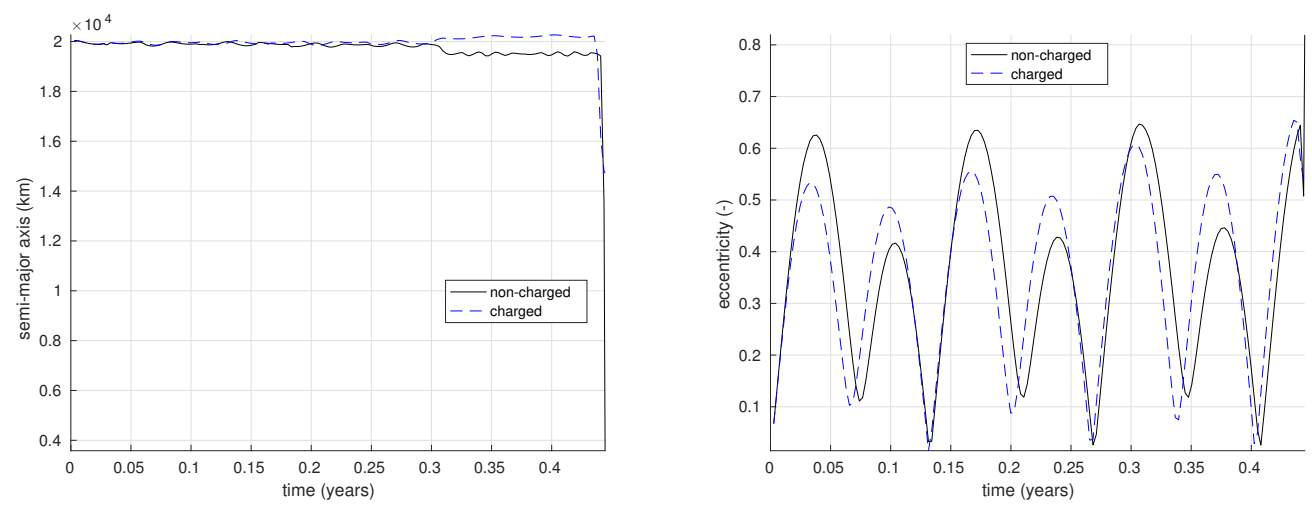

Fig. 19: Time history of semi-major axis and eccentricity dust particles
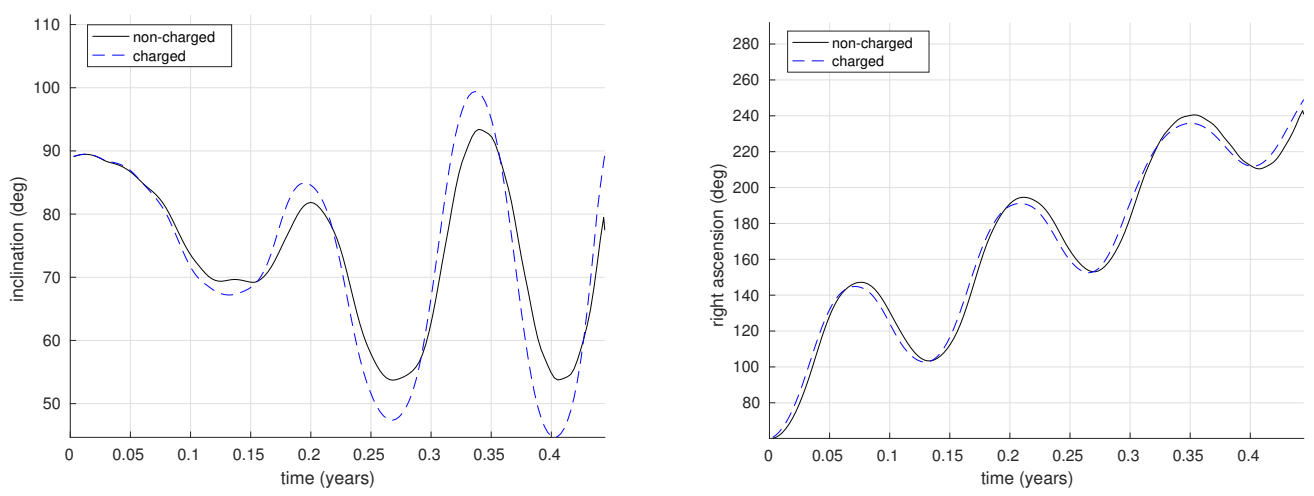

Fig. 20: Time history of inclination and right ascension of the ascending node for dust particles

\section{Conclusion}

An object orbiting the Earth and that passively acquires an electrostatic charge on its surface becomes subject to the magnetic force. The corresponding acceleration is proportional to the charge and orthogonal to both the magnetic field and the velocity relative to that field. The value of the charge is the product of two multiplicative parameters: electrostatic capacitance and surface 
potential. In LEO, both of these quantities mostly depend on the local electron temperature, while the capacitance is also dependent on the ambient Debye length which characterizes the plasma and is a function of both temperature and density. The profiles of these quantities in the ionosphere vary primarily according to magnetic latitude and local time, with secondary variations due to the local season. The additional consideration of photoelectron current adds a dependency on the exposure to sunlight. Due to the lack of a comprehensive model of the plasma environment in MEO, a simple modeling of the surface potential has been adopted in this study: positive in full exposure to sunlight, negative otherwise. It always turns out that the surface charge is proportional to the area-to-mass ratio. This means that the acceleration due to the Earth's magnetic field scales linearly with this parameter, similarly to atmospheric drag and radiation pressure. In this study, it was set to a value of $10 \mathrm{~m}^{2} / \mathrm{kg}$, which belongs to the upper boundary of the range for space debris.

This high area-to-mass ratio implies large values for all non-gravitational forces included in model, not only the magnetic one. When compared to other perturbations in LEO, the acceleration due to electrostatic charging remains several orders of magnitude smaller. In MEO, atmospheric drag does not have a relevant impact on the object dynamics, whereas the gravitational terms and the solar radiation pressure are the dominant perturbing accelerations. In this work, the impact of the Lorentz force on trajectories has been investigated for two different geometries. For a spherical object, the impact of electrostatic charging was shown to be fairly small. In LEO, re-entry occurs within decades and there is not enough time for the two trajectories to significantly diverge. Nonetheless, a coupling with radiation pressure seems to be caused by eclipse conditions. On the other hand in MEO, where there is no orbit decay, the magnitude of the magnetic force is much smaller than at lower altitudes, resulting in a negligible impact on the dynamics, even on the span of a century. Noticeable effects appear only when looking at much lighter objects than debris, such as dust particles that can actually re-enter or escape the Earth on short time scales due to their high susceptibility to non-gravitational forces.

With a square flat plate, the effects on orbital motion at low altitude are comparable to the spherical case. On the other hand, the magnetic torque induced by a displacement of the barycenter with respect to the center of symmetry of the plate can have a significant impact on attitude. While 
the changes in Keplerian coordinates are too small to create large shifts in re-entry times, the changes in attitude would for instance cause radically different conditions for the orientation at impact in the case of orbital collision.

Perspectives include looking at rare events such as auroral charging in LEO that can temporarily but significantly increase the magnitude of the surface charge. This short-term phenomenon could have a non-negligible impact on the orbital elements. Another topic of interest for attitude dynamics would be to study objects with non-uniform surface properties or asymmetric geometries, which directly induce a magnetic torque.

\section{Acknowledgment}

The authors would like to thank the United Kingdom Space Agency for the funding of the project entitled Debris Evolution Uncertainty Quantification which initiated this work.

\section{References}

[1] Hamilton, D. P., "Motion of dust in a planetary magnetosphere: Orbit-averaged equations for oblateness, electromagnetic, and radiation forces with application to Saturn's E ring," Icarus, Springer, Vol. 101, No. 2, 1993, pp. 244-264.

[2] Daquin J., Rosengren A.J., Alessi E.M., Deleflie F., Valsecchi G.B. and Rossi A., "The dynamical structure of the MEO region: long-term stability, chaos, and transport," Celestial Mechanics and Dynamical Astronomy, 2016, Vol. 124, No. 4, pp. 335-366.

[3] Anselmo, L. and Pardini, C., 'Long-term dynamical evolution of high area-to-mass ratio debris released into high earth orbits," Acta Astronautica, Vol. 67, No. 1, 2010, pp. 204-216.

[4] Valk, S. and Lemaître, A., "Semi-analytical investigations of high area-to-mass ratio geosynchronous space debris including EarthâĂŹs shadowing effects," Advances in Space Research, Vol. 42, No. 8, 2008, pp. 1429-1443.

[5] Pollock, G. E. and Gangestad, J. W. and Longuski, J. M., "Inclination change in low-Earth orbit via the geomagnetic Lorentz force," Journal of guidance, control, and dynamics, AIAA, Vol. 33, No. 5, 2010, pp. $1387-1395$.

[6] Peck, M., "Prospects and challenges for Lorentz-augmented orbits," AIAA guidance, navigation, and control conference and exhibit, 2005, pp. 5995.

[7] Leach, R. D. and Alexander, M. B., "Failures and anomalies attributed to spacecraft charging," 1995. 
[8] Paul, S. N. and Frueh, C., "Space Debris Charging and its Effect on Orbit Evolution," AIAA/AAS Astrodynamics Specialist Conference, 2016, pp. 5254.

[9] Hoshi, K. and Serra, R. and Vasile, M. and Yamakawa, H., "Study of the effects of the Lorentz force on space objects with high area-to-mass ratio," 31st International Symposium on Space Technology and Science, 2017.

[10] Walker M.J., Ireland B. and Owens J., "A set modified equinoctial orbit elements," Celestial Mechanics and Dynamical Astronomy, 1985, Vol. 36, No. 4, pp. 409-19.

[11] Montenbruck, O. and Gill, E., "Satellite orbits," Springer, Vol. 2, 2000, pp. 53-116.

[12] Leaton, B., "International geomagnetic reference field 1975," Eos, Transactions American Geophysical Union, Vol. 57, No. 3, 1976, pp. 120-121, Wiley Online Library.

[13] Scanlon, T. J. and Roohi, E. and White, C. and Darbandi, M. and Reese, J. M., "An open source, parallel DSMC code for rarefied gas flows in arbitrary geometries," Computers and Fluids, Vol. 39, No. 10, 2010, pp. 2078-2089.

[14] Vasile M., Minisci E., Serra R., Beck J. and Holbrough I., "Analysis of the de-orbiting and re-entry of space objects with high area to mass ratio," AIAA/AAS Astrodynamics Specialist Conference, Long Beach, California, 2016.

[15] Whipple, E. C., "Potentials of surfaces in space," Reports on Progress in Physics, Vol. 44, 1981, pp. 1197-1250.

[16] Katz, I., Mandell, M., C. Roche, J., and Purvis, C., "Secondary electron generation, emission and transport: effects on spacecraft charging and NASCAP models," Journal of Electrostatics, Vol. 20, 1987, pp. 109-121.

[17] Lai, S. T., "Fundamentals of spacecraft charging: spacecraft interactions with space plasma," Princeton University Press, 2011.

[18] Mott-Smith, H. M., and Langmuir, I., "Theory of Collectors in Gaseous Discharges," Physical Review, Vol. 28, 1926, pp. 727-763.

[19] Laframboise, J. G., and Parker, L. W., "Probe Design for Orbit-Limited Current Collection," Physics of Fluids, Vol. 16, No. 5, 1973, pp. 629.

[20] Allen, J. E., "Probe Theory - the Orbital Motion Approach," Physica Scripta, Vol. 45, No. 5, 1992, pp. 497-503.

[21] Lai, S. T., "An improved Langmuir probe formula for modeling satellite interactions with neargeostationary environment," Journal of Geophysical Research, Vol. 99, No. A1, 1994, pp. 459. 
[22] Nakagawa, T. and Ishii, T. and Tsuruda, K. and Hayakawa, H. and Mukai, T., "Net current density of photoelectrons emitted from the surface of the GEOTAIL spacecraft," Earth, planets and space, Vol. 52, No. 4, 2000, pp. 283-292.

[23] Gurnett D.A. and Bhattacharjee A., "Introduction to plasma physics," Press Syndicate of the University of Cambridge, 2005.

[24] Bilitza, D., "International reference ionosphere 1990. URSI/COSPAR," Report NSSDC/WDC-A-RछS 90-22, 1990.

[25] Booker H.G., 'Fitting of multi-region ionospheric profiles of electron density by a single analytic function of height," Journal of Atmospheric and Terrestrial Physics, 1977, Vol. 39, No. 5, pp. 619-23.

[26] Sarno-Smith, Lois K. and Larsen, B. A. and Skoug, R. M. and Liemohn, M. W. and Breneman, A. and Wygant, J. R. and Thomsen, M. F., "Spacecraft surface charging within geosynchronous orbit observed by the Van Allen Probes," Space Weather, Vol. 14, No. 2, 2016, pp. 151-164. 